\title{
Charge and spin edge currents in $2 d$ Floquet topological superconductors
}

\author{
P. D. Sacramento ${ }^{1,2}$ \\ 1 CeFEMA, Instituto Superior Técnico, Universidade de Lisboa, Av. Rovisco Pais, 1049-001 Lisboa, Portugal \\ ${ }^{2}$ Centro Brasileiro de Pesquisas Físicas, Rua Dr. Xavier Sigaud 150, Urca 22290-180, Rio de Janeiro, RJ, Brazil
}

(Dated: September 27, 2021)

\begin{abstract}
A time periodic driving on a topologically trivial system induces edge modes and topological properties. In this work we consider triplet and singlet superconductors subject to periodic variations of the chemical potential, spin-orbit coupling and magnetization, in both topologically trivial and nontrivial phases, and study their influence on the charge and spin currents that propagate along the edges of the two-dimensional system, for moderate to large driving frequencies. Currents associated with the edge modes are induced in the trivial phases and enhanced in the topological phases. In some cases there is a sign reversal of the currents as a consequence of the periodic driving. The edge states associated with the finite quasi-energy states at the edge of the Floquet zone are in general robust, while the stability of the zero quasi-energy states depends on the parameters. Also, the spin polarization of the Floquet spectrum quasi-energies is strong as for the unperturbed topological phases. It is found that in some cases the unperturbed edge states are immersed in a continuum of states due to the perturbation, particularly if the driving frequency is not large enough. However, their contribution to the edge currents and spin polarization is still significant.
\end{abstract}

PACS numbers: 74.40.Gh, 72.25.-b, 74.40.Kb

\section{INTRODUCTION}

Topological systems have attracted great interest ${ }^{12}$ and, specifically in the case of topological superconductors 3 , a great effort has been put towards prediction and detection of Majorana fermions ${ }^{4}$. The robustness of topological systems to perturbations is a key feature with wide potential applications, such as the edge state Majorana fermions as building blocks for topological quantum computation, due to their non-abelian statistics. It has been shown that topological systems are quite robust to a quantum quench, as exemplified by the toric code model[5]. It has also been shown recently that, in an infinite system, the Chern number can not be changed by a unitary evolution, even though it is possible to change the Bott index and topology if the system has a finite size ${ }^{7}$, as obtained earlier in the case of quenches in topological superconductors $^{8}$. Therefore, the response to a time dependent perturbation of the edge states in finite systems may not be protected by topology. Furthermore, quenches in superconducting systems with topological properties, performed self-consistently ${ }^{9}$, showed the importance of the topological properties in the evolution of the system $[10$ and raised questions regarding the survival of the topological order to the quench $5111-13$. So the issue is not resolved and is attracting considerable attention.

Several examples that host Majorana fermions as edge states are provided by several superconducting p-wave systems $\frac{14 \mid 15}{15}$ and various other systems that mix superconducting order (eventually by proximity effects) with Zeeman fields and/or spin-orbit coupling $\frac{16}{66}$ or tunneling of Cooper pairs 17. A recent proposal for the existence of localized Majorana edge states at the ends of a magnetic chain in contact with a conventional superconductor ${ }^{18}$ has been confirmed experimentally 19 (there is however some debate because of their unexpected very localized nature ${ }^{20 \mid 21}$ ) and it has been shown, combining topological with non-topological regions in wires, that it also provides a good basis of states to implement the braiding properties of the Majorana fermions 22 , with potential interest in quantum computation. The fermion exchange in a unidimensional system recently proposed ${ }^{23}$ is also possible to implement with a magnetic chain. Various other proposals have been presented for the existence of Majorana zero energy states including recently multiband systems 24 26, where the non-trivial interband coupling leads to interesting topological properties, as shown also in the context of topological Kondo insulators $27 / 28$.

In higher dimensions the edge modes are in general propagating in the directionss parallel to the edge and localized in the perpendicular direction. Their nature as protected states suggests the existence of spontaneous currents, either charge or spin currents, that have aroused considerable interest in several contexts such as in the context of spintronics, as a means to generate and manipulate spin currents. The anomalous Hall effect due to the presence of spin-orbit coupling has been considered 29 as well as the appearance of currents due to magnetic impurities 30132 . The study of the currents generated at interfaces between topological insulators and a supercondutor has been considered ${ }^{33 / 34}$ such as the charge and spin conductances. A manipulation using electrical means has been proposed ${ }^{\sqrt{35}}$ as well as their spin correlations using a gate potentia 36 . Chiral $p$-wave superconductors are expected to show spontaneous edge currents. Some other chiral systems were, however, shown to not have spontaneous currents $37[39$ but, with some admixtures of different pairings, these arise together with some non-trivial spin polarization 40 . Even though the topic has been studied for some time, the experimental verifi- 
cation of the spontaneous generation of edge currents is still a topic of considerable attention. It is expected that a strong candidate in the class of chiral p-wave superconductors $\mathrm{Sr}_{2} \mathrm{RuO}_{4}$ will exhibit spontaneous currents 4142 . Even though the material $\mathrm{Sr}_{2} \mathrm{RuO}_{4}$ has been shown to break time reversal symmetry due to the existence of the Kerr effect $\frac{43}{4}$, no edge surrents have been found $\underline{44} 45$ even though evidence for the edge states has been seen by in-plane tunneling spectroscopy 46 . Various proposals have been presented to explain the absence of the edge currents ${ }^{47}$ using the result of an effective high Chern number $\underline{48 \mid 49}$ or effects of disorder $\underline{38}$. Also, it has been proposed that the absence of the edge currents may be due to a spin singlet pairing instead of the generally assumed p-wave triplet pairing $\sqrt{50}$.

As mentioned above, it is interesting to study the robustness of the edge states to time dependent perturbations. In the context of the Creutz ladder, it was shown that the presence of edge states modifies the process of defect production expected from the Kibble-Zurek mechanism, leading in this problem to a scaling with the change rate with a non-universal critical exponent ${ }^{51}$. A similar result was obtained for the one-dimensional superconducting Kitaev model, where it was shown that, although bulk states follow the Kibble-Zurek scaling, the produced defects for an edge state quench are quite anomalous and independent of the quench rate ${ }^{8152}$. The behavior of edge states under an abrupt quantum quench has also been considered very recently in the context of a two-dimensional topological insulator ${ }^{53}$, where it was found that, in the sudden transition from the topological insulator to the trivial insulator phase, there is a collapse and revival of the edge states 54 . Similar results were obtained for the one-dimensional Kitaev model 55 , and also studying the signature of the Majoranas in the entanglement spectrum 56 . Their dynamical formation and manipulation has been considered in ${ }^{57}$ and ${ }^{11}$. The effect of a sudden quench of the parameters of the Hamiltonian of a two-dimensional triplet superconductor has also been studied, and the robustness of the edge states was considered $\sqrt{8}$. In general it was found that the edge states decay due to the quench, even though in some cases they are quite robust, such as in the case of weak spin-orbit coupling, or when there are matching momentum states in the initial and final states. The effect of parity blocking on the dynamics of the edge modes has been considered recently in which case the dynamics is restricted if there is a change in fermion parity accross

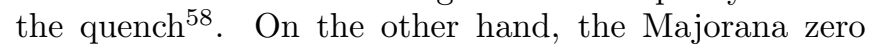
modes lead to some universal non-equilibrium signature in the Loschmidt echo with an universal exponent associated with the algebraic decay $\sqrt{59}$. Also, the dynamics of the tunneling into non-equilibrium edge states has been proposed as a possible signature of the existence of these states 60 . Non-equilibrium situations also may allow the transport of Majorana edges states using extended gapless regions with a small but finite overlap with the Majoranas 61 . Their effect has also been considered in $\frac{62}{\text {. }}$
While quenches, either abrupt or slow, in general destabilize the edge states, topological phases can be induced by periodically driving the Hamiltonian of a nontopological system, such as shown before in topological insulators 65 and in topological superconductors, with the appearance of Majorana fermions 66, 69. Their appearance in a one-dimensional p-wave superconductor was studied in Ref!70 and in Ref!71 introducing external periodic perturbations; the case of intrinsic periodic modulation was also considered 72 . The periodic driving leads to new topological states 64 , and to a generalization of the bulk-edge correspondence, that reveals a richer structure $73 / 74$ as compared with the equilibrium situation 75/76. Similarly, in topological superconductors new phases may be induced and manipulated due to the presence of the periodic driving $70 / 77 / 78$.

In general the problem is complex due to problem of dissipation through coupling to baths. Their effect have recently been considered and their detection has been proposed using transport properties $79 \sqrt{81}$, as well as magnetization signatures using the magnetic fields the currents should produce 82 .

In this work we compare the charge and spin edge currents of the unperturbed Hamiltonian with those of the perturbed Hamiltonian for triplet superconductors. We consider a system with no coupling to its environment except for the coupling to the periodic perturbation. To simplify, we consider perturbations where only one of the parameters of the Hamiltonian is periodicaly changed, such as the hopping, spin-orbit coupling, chemical potential or magnetization. We mainly focus on the last two parameter changes since are easier to implement experimentally. In section II we review the Hamiltonian considered and in section III we review the theory of the Floquet states that result from a periodic perturbation. In section IV we illustrate the method by presenting solutions of the quasi-energies for different cases, as a function of the perturbation amplitude and frequency, and verify the convergence of the truncation procedure of the Hamiltonian matrix of the Floquet problem. In section $\mathrm{V}$ we present results for the edge currents and the spin polarization of the states of system. We conclude with section VI.

\section{UNPERTURBED TOPOLOGICAL SUPERCONDUCTOR}

We consider two-dimensional superconductors that display topological phases. Standard examples are triplet superconductors with $p$-wave symmetry. We also consider the presence of spin-orbit coupling and a Zeeman term (magnetization that may be due to proximity effect or intrinsic). In the case of a non-centrosymmetric system, since parity is no longer conserved, a spin singlet pairing is also possible. Therefore we also consider an admixture of a conventional $s$-wave component. Even without a triplet pairing component a conventional su- 
perconductor may also display topological properties in the presence of magnetization.

The model considered here was studied in various refserences before such as in Refs.16131. We write the Hamiltonian for the bulk system as

$\hat{H}=\frac{1}{2} \sum_{\boldsymbol{k}}\left(\boldsymbol{\psi}_{\boldsymbol{k}}^{\dagger}, \boldsymbol{\psi}_{-\boldsymbol{k}}\right)\left(\begin{array}{cc}\hat{H}_{0}(\boldsymbol{k}) & \hat{\Delta}(\boldsymbol{k}) \\ \hat{\Delta}^{\dagger}(\boldsymbol{k}) & -\hat{H}_{0}^{T}(-\boldsymbol{k})\end{array}\right)\left(\begin{array}{c}\boldsymbol{\psi}_{\boldsymbol{k}} \\ \boldsymbol{\psi}_{-\boldsymbol{k}}^{\dagger}\end{array}\right)(1)$

where $\left(\boldsymbol{\psi}_{\boldsymbol{k}}^{\dagger}, \boldsymbol{\psi}_{-\boldsymbol{k}}\right)=\left(\psi_{\boldsymbol{k} \uparrow}^{\dagger}, \psi_{\boldsymbol{k} \downarrow}^{\dagger}, \psi_{-\boldsymbol{k} \uparrow}, \psi_{-\boldsymbol{k} \downarrow}\right)$ and

$$
\hat{H}_{0}=\epsilon_{\boldsymbol{k}} \sigma_{0}-M_{z} \sigma_{z}+\hat{H}_{R} .
$$

Here, $\epsilon_{\boldsymbol{k}}=-2 \tilde{t}\left(\cos k_{x}+\cos k_{y}\right)-\mu$ is the kinetic part, $\tilde{t}$ denotes the hopping parameter set in the following as the energy scale $(\tilde{t}=1), \mu$ is the chemical potential, $\boldsymbol{k}$ is a wave vector in the $x y$ plane, and we have taken the lattice constant to be unity. Furthermore, $M_{z}$ is the Zeeman splitting term responsible for the magnetization, in $\tilde{t}$ units. The Rashba spin-orbit term is written as

$$
\hat{H}_{R}=\boldsymbol{s} \cdot \boldsymbol{\sigma}=\alpha\left(\sin k_{y} \sigma_{x}-\sin k_{x} \sigma_{y}\right),
$$

where $\alpha$ is measured in the same units. The matrices $\sigma_{x}, \sigma_{y}, \sigma_{z}$ are the Pauli matrices acting on the spin sector, and $\sigma_{0}$ is the $2 \times 2$ identity. The pairing matrix reads 83

$$
\hat{\Delta}=i(\boldsymbol{d} \cdot \boldsymbol{\sigma}) \sigma_{y}=\left(\begin{array}{cc}
-d_{x}+i d_{y} & d_{z}+\Delta_{s} \\
d_{z}-\Delta_{s} & d_{x}+i d_{y}
\end{array}\right) .
$$

where $\Delta_{s}$ is the symmetric part and the vector $\boldsymbol{d}$ parametrizes the anti-symmetric part.

The energy eigenvalues and eigenfunction may be obtained solving the Bogoliubov-de Gennes equations

$$
\left(\begin{array}{cc}
\hat{H}_{0}(\boldsymbol{k}) & \hat{\Delta}(\boldsymbol{k}) \\
\hat{\Delta}^{\dagger}(\boldsymbol{k}) & -\hat{H}_{0}^{T}(-\boldsymbol{k})
\end{array}\right)\left(\begin{array}{c}
u_{n} \\
v_{n}
\end{array}\right)=\epsilon_{\boldsymbol{k}, n}\left(\begin{array}{c}
u_{n} \\
v_{n}
\end{array}\right)
$$

The 4-component spinor can be written as

$$
\left(\begin{array}{c}
u_{n} \\
v_{n}
\end{array}\right)=\left(\begin{array}{c}
u_{n}(\boldsymbol{k}, \uparrow) \\
u_{n}(\boldsymbol{k}, \downarrow) \\
v_{n}(-\boldsymbol{k}, \uparrow) \\
v_{n}(-\boldsymbol{k}, \downarrow)
\end{array}\right) .
$$

We consider either a superconductor with $\boldsymbol{d}=$ $d\left(\sin k_{y},-\sin k_{x}, 0\right)$ or $\boldsymbol{d}=d_{z}\left(0,0, \sin k_{x}-i \sin k_{y}\right)$ with or without a contribution from the local s-wave pairing $\Delta_{s}$. The first case applies if the spin-orbit is strong. In this case the pairing is aligned $\frac{84}{40}$ along the spin-orbit vector $\boldsymbol{s}$. This case is denoted by strong coupling case. Relaxing this restriction allows that the two vectors are not aligned. This case is denoted by weak spin-orbit coupling and has been considered before in the context of the anomalous Hall effect and the calculation of the Hall conductance $30 \mid 31$. In the strong-coupling case $\boldsymbol{d}=\left(d_{x}, d_{y}, d_{z}\right)=(d / \alpha) s$. The second case, usually called $p+i p$ superconductor, has been the focus of great attention since it has spontaneous chiral states due to its

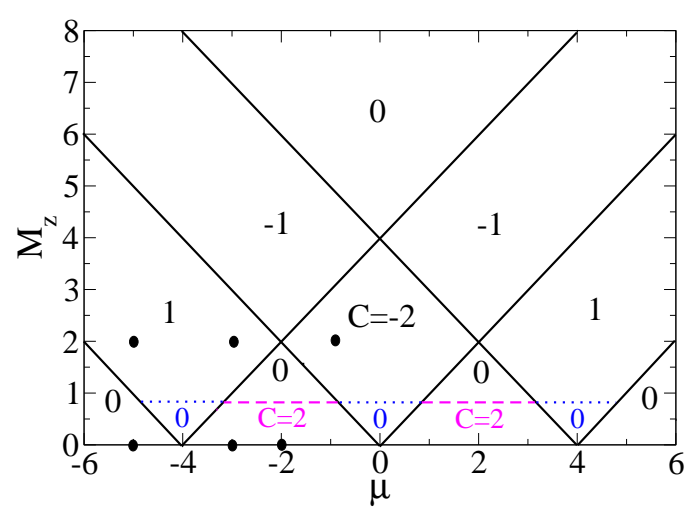

FIG. 1: (Color online) Phase diagram for the spin triplet superconductor with $\boldsymbol{d}=\left(d_{x}, d_{y}, 0\right)$. The phase diagramfor a $s$-wave superconductor is very similar except in the vicinity of zero magnetization (limited approximately by the dotted lines) where the system is topologically trivial. In the case of a triplet superconductor with $\boldsymbol{d}=\left(0,0, d_{z}\right)$ with a complex $z$-component, the phase diagram is also similar with the exception of a region around zero magnetic field (limited by dashed lines) that is topological, with Chern number 2.

time-reversal breaking structure. In contrast, in the absence of the Zeeman term the first case does not break time-reversal symmetry (TRS) and in the topological phases the edge states have a helicoidal structure. The system then belongs to the symmetry class DIII where the topological invariant is a $\mathbb{Z}_{2}$ index ${ }^{75}$. If the Zeeman term is finite or in the case of the $p+i p$ pairing, TRS is broken and the system belongs to the symmetry class D. The topological invariant that characterizes this phase is the first Chern number $C$, and the system is said to be a $\mathbb{Z}$ topological superconductor.

The Chern number is obtainable as an integral of the Berry curvature over the Brillouin zone ${ }^{85 \mid 86}$. Summing over the occupied bands the Chern number has been calculated $16 / 31$. It is convenient to calculate the Chern number by computing the flux of the Berry curvature over plaquetes in the Brillouin zone 86 .

The results in the parameter space for the various pairing symmetries are shown in Fig. 1 using the parameters $\alpha=0.6, d=0.6$ and in the case of strong spin-orbit coupling. In this case, changing these parameters only leads to a quantitative change of the shape of the energy bands and no qualitative changes are observed in the topological properties. As shown before ${ }^{16}$, the spin-orbit coupling does not change the topology. The phase diagram for the case of a $s$-wave superconductor is very similar except for values of the magnetization smaller than the amplitude of $\Delta_{s}$. In these regimes the Chern number vanishes and the system is topologically trivial for all values of the chemical potential. Something similar happens in the case of the $p+i p$ superconductor in the sense that some regimes at values of the magnetization smaller 
than the pairing amplitude, $d_{z}$, the Chern number is different from the case of the $d_{x}, d_{y}$ pairing. In these regimes it is finite, $C=2$, due to the broken TRS, even in the absence of a finite magnetization. However, for some values of the chemical potential, a finite magnetization will change the Chern number to zero (even though there are unprotected edge states).

Due to the bulk-edge correspondence if the system is placed in a strip geometry and the system is in a topologically non-trivial phase, there are robust edge states, in a number of pairs given by the Chern number, if time reversal symmetry is broken. There are also counterpropagating edge states in the $Z_{2}$ phases even though the Chern number vanishes, as in the spin Hall effect. In these phases time reversal symmetry is preserved and the Kramers pairs of edge states give opposite contributions to the Chern number. Interestingly, turning on the magnetization (Zeeman field) time reversal symmetry is broken and the edge states are no longer topologically protected. However, it was found that, even in regimes where $C=0$, there are edge states, reminiscent of the edge states of the $Z_{2}$ phases, as long as the chemical potential satisfies $|\mu|<4 \tilde{t}$. The regimes where the magnetization is zero and $|\mu|>4 \tilde{t}$ are topologically trivial and in a strip geometry have no edge states.

\section{PERIODIC DRIVING}

The time evolution of a state under the influence of a time dependent Hamiltonian is given by

$$
i \frac{\partial}{\partial t} \psi(k, t)=H(k, t) \psi(k, t)
$$

where $k$ is the momentum, $t$ the time and $\hbar=1$. We can decompose the Hamiltonian in two terms a time independent one, $H(k)$, and an extra term due to the external time-dependent perturbation, that we want to take as periodic with a given frequency, $\omega$,

$$
H(k, t)=H(k)+f(\omega t) H_{d}(k)
$$

Here $H_{d}(k)$ is of the form of the unperturbed Hamiltonian but with only one non-vanishing term. Looking for a solution of the type

$$
\psi(k, t)=e^{-i \epsilon(k) t} \Phi(k, t)
$$

and using that $\Phi(k, t)=\Phi(k, t+T)$, where $T$ is the period $(\omega=2 \pi / T)$ and $f(\omega t)=f(\omega(t+T))$, one gets that

$$
\left(H(k, t)-i \frac{\partial}{\partial t}\right) \Phi(k, t)=\epsilon(k) \Phi(k, t)
$$

The time-independent quasi-energies $\epsilon(k)$ are the eigenvalues of the operator $H(k, t)-i \frac{\partial}{\partial t}$ and the function $\Phi(k, t)$ the eigenfunction. Since this function is periodic, we can expand it as

$$
\Phi(k, t)=\sum_{m} \phi_{m}(k) e^{i m \omega t}
$$

Inserting this expansion in equation we obtain the eigensystem

$$
\sum_{m^{\prime}} H_{m m^{\prime}}(k) \phi_{m^{\prime}}(k)=\epsilon(k) \phi_{m}(k)
$$

The Hamiltonian matrix is given by

$$
H_{m m^{\prime}}(k)=\delta_{m m^{\prime}} m \omega+\frac{1}{T} \int_{0}^{T} d t e^{-i m \omega t} H(k, t) e^{i m^{\prime} \omega t}
$$

Choosing a perturbation of the type $f(\omega t)=\cos (\omega t)$ the second term of the Hamiltonian matrix reduces to $1 / 2\left(\delta_{m^{\prime}+1, m}+\delta_{m^{\prime}-1, m}\right)$.

The time evolution of the state is then obtained solving for the quasi-energies, $\epsilon(k)$, and the functions $\phi_{m}(k)$ diagonalizing the infinite matrix

$$
\left(\begin{array}{ccccccc}
\cdots & \ldots & \ldots & \ldots & \ldots & \ldots & \ldots \\
\cdots & (m-2) \omega+H(k) & \frac{1}{2} H_{d}(k) & 0 & 0 & 0 & \ldots \\
\cdots & \frac{1}{2} H_{d}(k) & (m-1) \omega+H(k) & \frac{1}{2} H_{d}(k) & 0 & 0 & \ldots \\
\cdots & 0 & \frac{1}{2} H_{d}(k) & m \omega+H(k) & \frac{1}{2} H_{d}(k) & 0 & \ldots \\
\cdots & 0 & 0 & \frac{1}{2} H_{d}(k) & (m+1) \omega+H(k) & \frac{1}{2} H_{d}(k) & \ldots \\
\ldots & 0 & 0 & 0 & \frac{1}{2} H_{d}(k) & (m+2) \omega+H(k) & \ldots \\
\ldots & \ldots & \ldots & \ldots & \cdots & \cdots & \ldots
\end{array}\right)
$$

The matrix can be reduced if the frequency is high enough and only a few values of $m$ are needed. The photon-dressing of the band structures due to the mixing of the bands is the important effect we consider $\frac{87}{87}$ We will neglect here any photon emission/absorption processes that affect the occupation numbers of the elec- 
trons. Equivalently one may consider that the photons are off-resonance. The effects of creation/absorption of photons if the driving frequency is not large enough were discussed befor ${ }^{80}$. The matrix $H_{d}(k)$ will be chosen as the unperturbed Hamiltonian, where only one of the parameters, hopping, chemical potential, spin-orbit coupling or magnetization will be considered to vary with time. The first three parameters preserve time reversal symmetry while the magnetization naturally breaks time reversal symmetry if the unperturbed Hamiltonian is in a regime with vanishing magnetization. Emphasis will be placed on the effects of varying the chemical potential or the magnetization which are easilly tuned externally. In this last case it has been determined before ${ }^{71}$ that even though the low energy states have a very low energy, they may not be strictly Majorana fermions since the eigenvalues of the Floquet operator (time evolution operator over one time period) are not strictly \pm 1 .

Due to the periodicity of the eigenfunctions, $\Phi(k, t+$ $T)=\Phi(k, t)$, the action of the evolution operator, $\mathcal{U}(t)$, on a state over a period, $T$, leads to the same state minus a phase

$$
|\psi(T)\rangle=\mathcal{U}(T)|\psi(0)\rangle=e^{-i \epsilon T}|\psi(0)\rangle
$$

Therefore, the quasi-energies are defined minus a shift of a multiple of $w=2 \pi / T$, and we can restrict the quasienergies to the first Floquet zone, defined by the interval $-w / 2 \leq \epsilon \leq w / 2$. States with quasi-energies $\epsilon=w / 2$ and $\epsilon=-w / 2$ are therefore equivalent and there is a reflection of any bands as one exits the Floquet zone from above (or below) and as one enters from below (or above). Considering the particle-hole symmetry of a superconductor, $\gamma_{-\epsilon}=\gamma_{\epsilon}^{\dagger}$ and the equivalence between the energies $\epsilon=-w / 2, w / 2$ one expects a new type of Majorana mode in addition to any zero energy states, the usual Majorana modes.

For the large enough driving frequencies considered here, the frequency description considered is particularly convenient. In the regime of small driving frequencies a time description is more convenient.

\section{ENERGY BANDS AND QUASI-ENERGIES}

The energy spectra of the unperturbed superconductors both in the trivial and in the topological phases have been determined before. The solutions for the quasienergies of the perturbed Hamiltonians lead to bands that have a similar structure. Here we focus on the energy and quasi-energy spectra in a ribbon geometry to give evidence to the states along the edges of the system.

At large frequencies, $w>4 \tilde{t}$, the size of the truncated matrix is relatively small and the quasi-energies and physical properties (calculated over the first Floquet zone) converge fast for small values of $m$. Considering $m=0$ one reproduces the Hamiltonian of the unperturbed superconductor. The first approximation for the driven system is obtained considering $m=1,0,-1$, then $m=2,1,0,-1,-2$ and so on. One may therefore use a short notation for the number of terms considered in the diagonalization of the Hamiltonian matrix by using $l=0,1,2,3, \cdots$. The unperturbed case is denoted by $l=0$ and the perturbed cases by $l=1,2, \cdots$ (considering that we are using $2 l+1$ states). If the frequency $w$ is small, one needs to consider large values of $l$ and the problem of finding the edge states in a ribbon geometry quickly becomes heavy computationally. Increasing the value of the frequency it is easy to find that it is enough to consider $l=2$, since taking $l=3$ leads to very similar results, with a good accuracy.

To illustrate the structure of the quasi-energies we consider first the case of $l=1$ and a $d_{x}, d_{y}$ superconductor in a topologically trivial phase. The results are shown in Figs. 2 and 3 where we consider periodic drivings in the hopping and in the magnetization for moderate couplings of $\tilde{t}_{d}=M_{z d}=2$. We consider frequencies $w=4,6,8,12$. As the frequency increases edge states appear at the border of the Floquet zone around $w / 2$ (and $-w / 2$ ). As the frequency increases further there is a continuum of states that fills the gap around zero energy. On the other hand, at higher energies close to the Floquet zone edge, as the frequency increases the edge states appear on top of the continuum and prevail as the gap around $w / 2$ opens. Therefore, as a consequence of the periodic driving edge states appear due to the Floquet spectrum in a system that is trivial if unperturbed. The edge states around $w / 2$ visible for the higher frequencies are robust and converge as $l$ increases. The two type of perturbations couple to states differently particularly in the spin structure. Also, the hopping preserves time reversal symmetry while the magnetization breaks time reversal symmetry. However, their effects at this order are very similar with respect to the edge states. The main difference is the response of the bulk states to the two types of perturbations, particularly seen at moderate frequencies where in the case of the magnetization perturbation the continuum appears at the energies where the edge states appear, even though at different momenta.

In Fig. 4 we show the convergence of the truncation procedure of the Hamiltonian matrix for a high value of the frequency $w=6$. We show results for $l=1,2,3$ and see that the results for $l=2$ and $l=3$ are very similar. For a smaller frequency value, $w=4$, the convergence is also fast. Moreover, for the set of parameters considered, with a relatively high coupling of $M_{z d}=4$ we see that, in addition to a fast convergence and effectiveness of the truncation procedure, besides the edge Majorana states close to the Floquet boundary robust Majoranas also appear at zero energy. For smaller frequencies and weak couplings the convergence is in general slower. As a consequence, and to reduce the computational effort, we consider a frequency $w=6$ in most results and limit the results to $l=2$.

It is also interesting to determine the effect of the periodic driving when the system is originally in a nontrivial topological phase. In Fig. 5 we show the effect of driving 

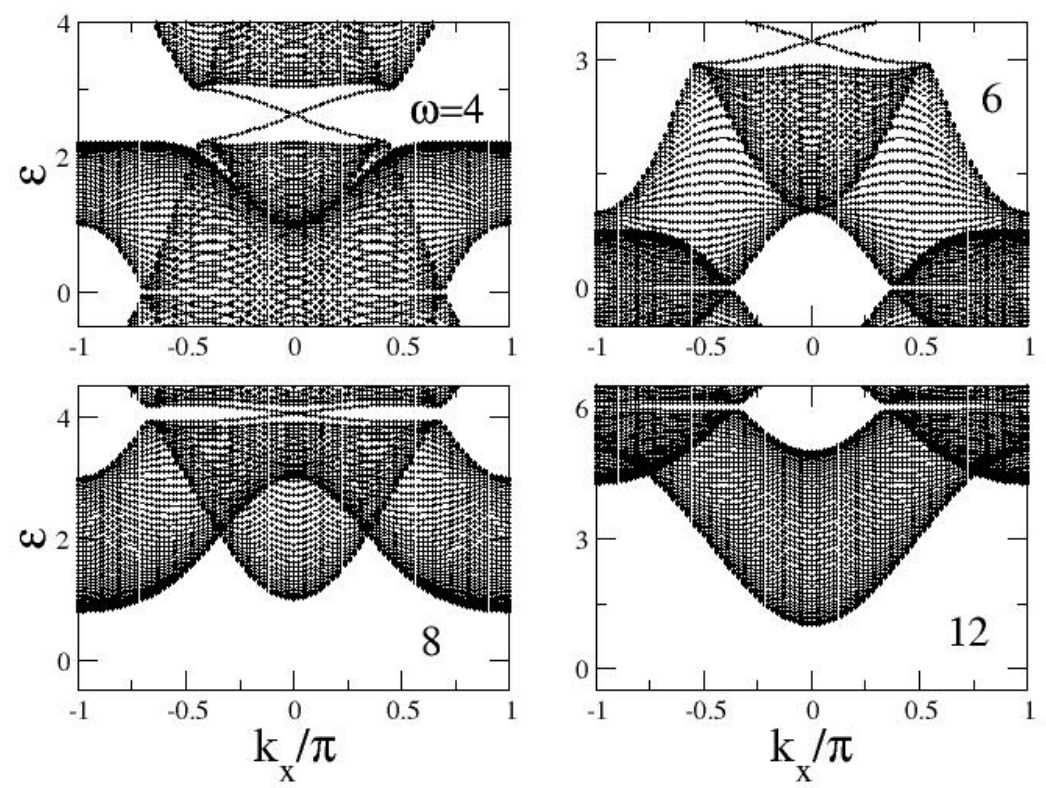

FIG. 2: Floquet spectra for $l=1$ for a $d_{x}, d_{y}$ triplet superconductor in a topologically trivial phase with $\alpha=0, M_{z}=0, \mu=$ $-5, \Delta_{s}=0, d_{z}=0, d=0.6$ where the hopping is changed with time with different frequencies $w=4,6,8,12$. The periodic driving is $t_{d} \cos w t$ with $t_{d}=2$. Note that the spectrum is symmetric around zero quasi-energy.
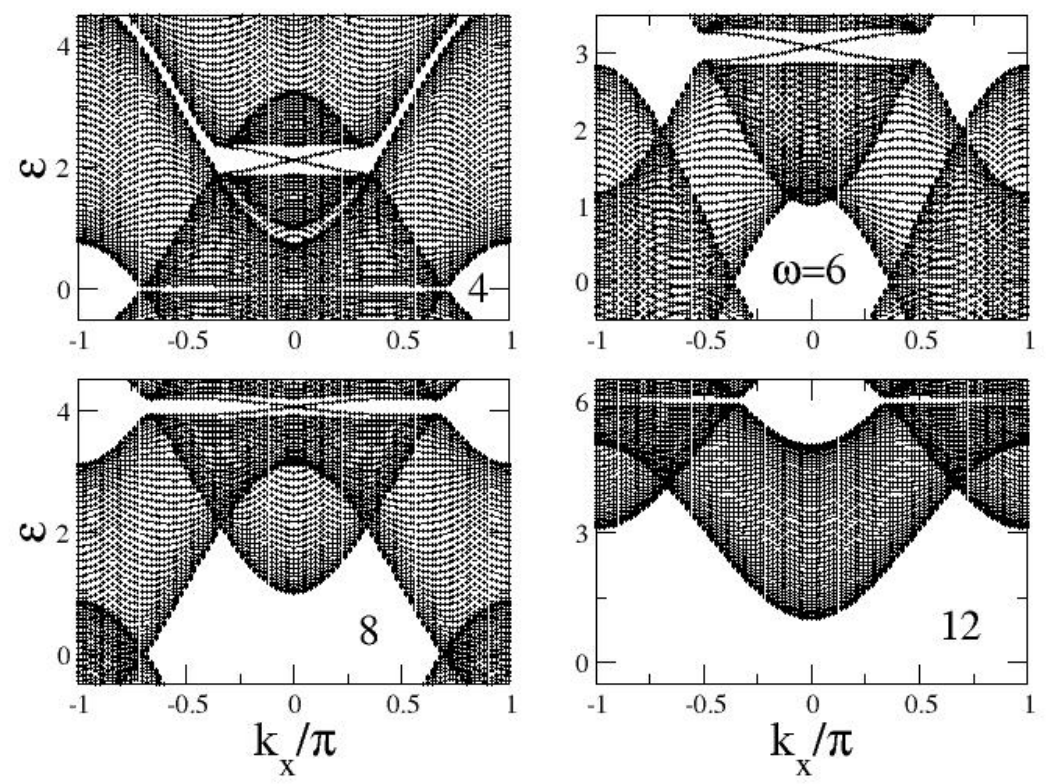

FIG. 3: Floquet spectra for $l=1$ for a $d_{x}, d_{y}$ triplet superconductor in a topologically trivial phase with $\alpha=0, M_{z}=0, \mu=$ $-5, \Delta_{s}=0, d_{z}=0, d=0.6$ where the magnetization is changed with time with different frequencies $w=4,6,8,12$. The periodic driving is $M_{z d} \cos w t$ with $M_{z d}=2$. 

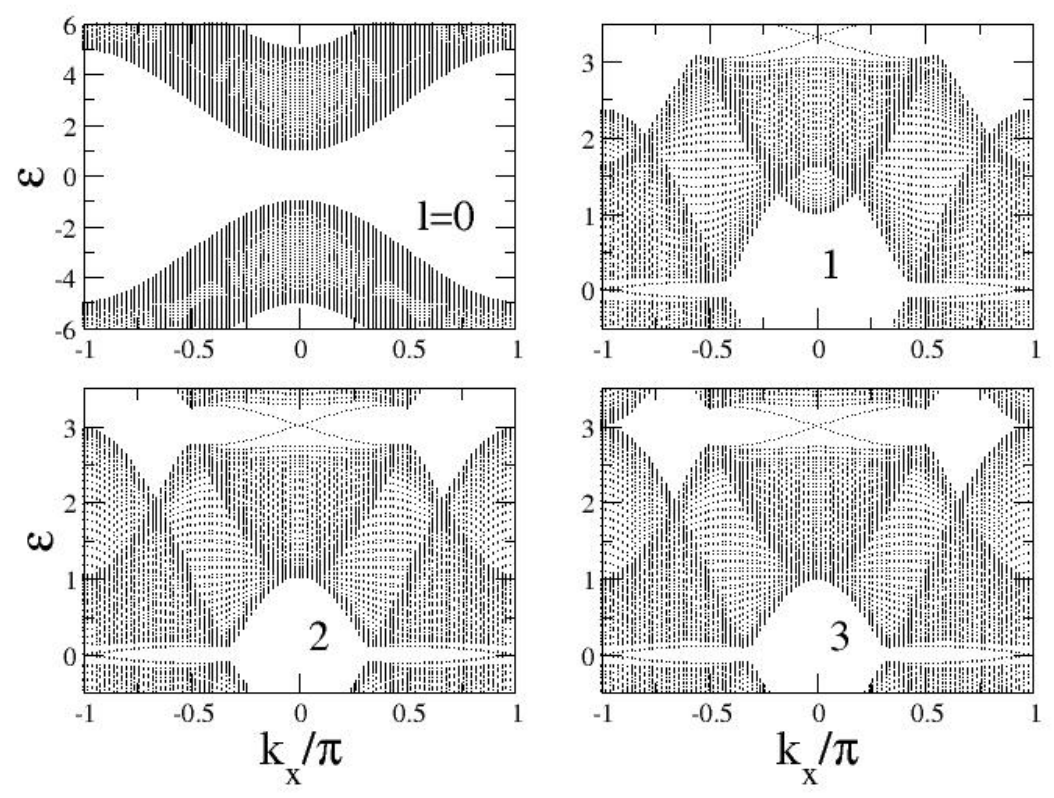

FIG. 4: Evolution of Floquet spectra for various values of $l, l=0,1,2,3$ for a $d_{x}, d_{y}$ triplet superconductor in a topologically trivial phase $\alpha=0.1, M_{z}=0, \mu=-5, \Delta_{s}=0.1, d_{z}=0, d=0.6$ where the magnetization is changed with time with frequency $w=6$. The periodic driving is $M_{z d} \cos w t$ with $M_{z d}=4$. The case $l=0$ is the unperturbed Hamiltonian. The results show the stability of two sets of Majorana modes both at zero energy and at the limit of the Floquet zone.
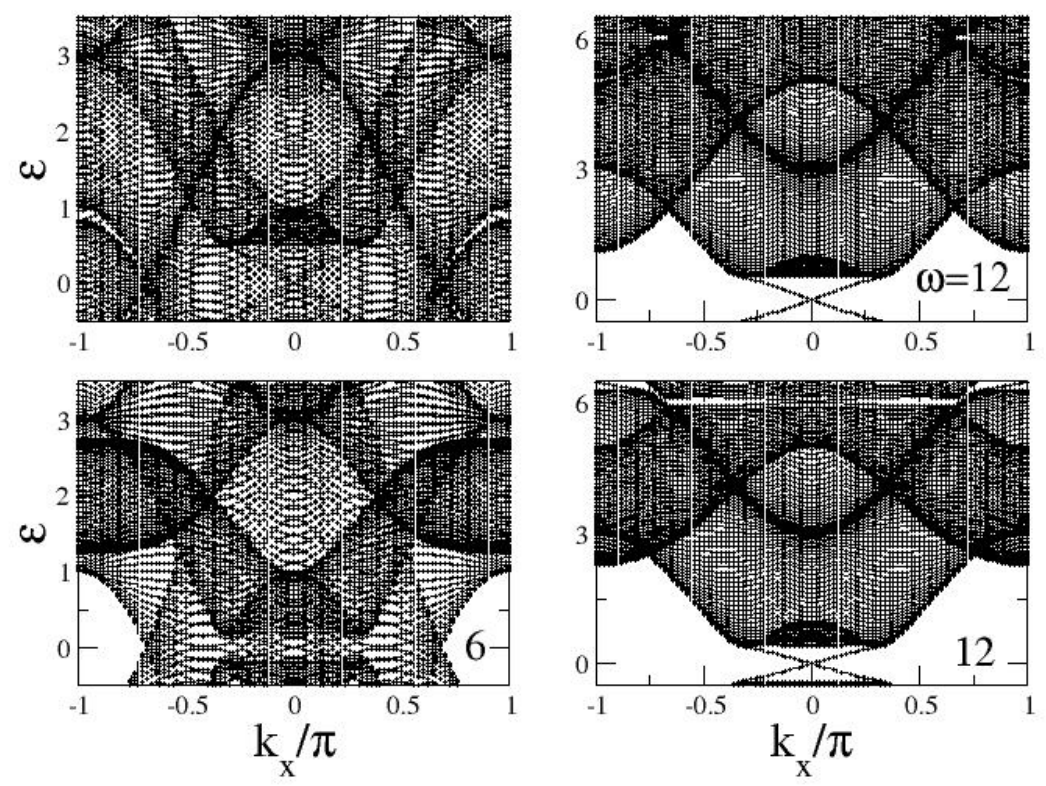

FIG. 5: Evolution of Floquet spectra for $l=1$ for a $d_{x}, d_{y}$ triplet superconductor in a topologically non-trivial phase $\alpha=0, M_{z}=2, \mu=-5, \Delta_{s}=0, d_{z}=0, d=0.6$ where the hopping is changed with time with frequencies $w=6,12$. The periodic driving is $\tilde{t}_{d} \cos w t$ with $\tilde{t}_{d}=0.5$ (top row) and $\tilde{t}_{d}=2$ (bottom row). 
the hopping with different frequencies $w=6,12$. The results are shown for $l=1$. We find that the continuum fills completely the gap, as illustrated in the case of $w=6$. Also, the same happens at the edge of the Floquet zone, particularly if the coupling is small $\tilde{t}_{d}=0.5$. Increasing the coupling to $\tilde{t}_{d}=2$ an edge state emerges at the boundary of the Floquet zone. Also both for moderate $(w=6)$ and high frequencies $(w=12)$ edge states appear near zero energy. However, the influence of the bulk states is considerable and at moderate frequency $w=6$ they are superimposed on the edge states.

The topology of the system in the presence of the periodic driving may also be considered in terms of the system in a torus. As mentioned above, the classification is richer and the bulk-edge correspondance is more subtle. It has been proposed ${ }^{73}$ that one may calculate the Chern number of the occupied quasi-energy bands, as for the unperturbed system. This works if there are clear gaps between the quasi-energy bands. In the context of this work, this will work in general at high frquencies. In most other cases, even though the analysis in the stripe geometry reveals edge states, these are often mixed with continuum states that close the gaps between bands, preventing the calculation of the Chern number of these bands. Also, due to the periodicity in Floquet quasi-energy space, we may have bands with Chern number that vanishes if the number of edge modes that enter the band equals the number of modes that leave it ${ }^{73}$.
Since our aim here are the currents associated with the edge modes, it is more enlightening to look at the band structure in the stripe geometry, since both the edge and the bulk states (projected along one spatial direction) are found.

\section{CURRENTS}

We consider a finite system of dimensions $N_{x} \times N_{y}$. We apply periodic boundary conditions along the $x$ direction and use a momentum representation and open boundary conditions along the transverse direction, $y$, solving the problem in a ribbon of width $N_{y}$. Writing

$$
\psi_{k_{x}, j_{y}, \sigma}=\frac{1}{\sqrt{N_{x}}} \sum_{j_{x}} e^{-i k_{x} j_{x}} \psi_{j_{x}, j_{y}, \sigma}
$$

we may rewrite the Hamiltonian matrix in terms of these operators. The diagonalization of this Hamiltonian involves the solution of a $\left(4 N_{y}\right) \times\left(4 N_{y}\right)$ eigenvalue problem for each momentum $k_{x}$. The energy states include states in the bulk and states along the edges. The eigenstates give us directly the wave functions in real space.

In this representation the states are column vectors that with no external time dependent perturbation is of the type

$$
\left(u\left(k_{x}, 1, \uparrow\right) u\left(k_{x}, 1, \downarrow\right) v\left(-k_{x}, 1, \uparrow\right) v\left(-k_{x}, 1, \downarrow\right) \cdots u\left(k_{x}, N_{y}, \uparrow\right) \quad u\left(k_{x}, N_{y}, \downarrow\right) v\left(-k_{x}, N_{y}, \uparrow\right) \quad v\left(-k_{x}, N_{y}, \downarrow\right)\right)^{T}
$$

The time evolved states due to the perturbation are the result of the diagonalization of the truncated Floquet matrix and each component $(\mathcal{U}=u$ or $\mathcal{U}=v)$ is of the form

$$
\mathcal{U}\left(k_{x}, j_{y}, \sigma, t\right)=\sum_{m} e^{i m \omega t} \phi_{m}\left(k_{x}, j_{y}, \sigma\right)
$$

The charge current operator along direction $x$ at a given position $\hat{j}_{y}$ along $y$ is given by

$\hat{j}_{c}\left(j_{y}\right)=\frac{2 e}{\hbar} \sum_{k_{x}} \boldsymbol{\psi}_{k_{x}, j_{y}}^{\dagger}\left(\begin{array}{cc}-\tilde{t} \sin \left(k_{x}\right) & -\frac{i}{2} \alpha \cos \left(k_{x}\right) \\ \frac{i}{2} \alpha \cos \left(k_{x}\right) & -\tilde{t} \sin \left(k_{x}\right)\end{array}\right) \boldsymbol{\psi}_{k_{x}, j_{y}}$

where $\boldsymbol{\psi}_{k_{x}, j_{y}}^{\dagger}=\left(\psi_{k_{x}, j_{y}, \uparrow}^{\dagger}, \psi_{k_{x}, j_{y}, \downarrow}^{\dagger}\right)$. The current has contributions from the hopping and the spin-orbit terms.

One may also define a longitudinal spin current, $\hat{j}_{s}\left(j_{y}\right)$, taking the difference between the two diagonal components of the charge current. The other terms correspond to spin-flip terms and do not contribute to the $z$ component of the spin current.

The average value of the charge current in the groundstate is given by summing over the single particle occu- pied states (negative energies) in the usual way

$$
\begin{array}{r}
j_{c}\left(j_{y}\right)=\left\langle\hat{j}_{c}\left(j_{y}\right)\right\rangle=\sum_{k_{x}, n} \\
\left\{\tilde { t } \operatorname { s i n } k _ { x } \left[\tilde{v}_{n}\left(-k_{x}, j_{y}, \uparrow\right) \tilde{v}_{n}^{*}\left(-k_{x} . j_{y}, \uparrow\right)\right.\right. \\
\left.+\tilde{v}_{n}\left(-k_{x}, j_{y}, \downarrow\right) \tilde{v}_{n}^{*}\left(-k_{x} . j_{y}, \downarrow\right)\right] \\
-\frac{i \alpha}{2} \cos k_{x}\left[\tilde{v}_{n}\left(-k_{x}, j_{y}, \uparrow\right) \tilde{v}_{n}^{*}\left(-k_{x} . j_{y}, \downarrow\right)\right. \\
\left.\left.-\tilde{v}_{n}\left(-k_{x}, j_{y}, \downarrow\right) \tilde{v}_{n}^{*}\left(-k_{x} \cdot j_{y}, \uparrow\right)\right]\right\}
\end{array}
$$

Here the functions are of the type

$$
\tilde{u}_{n}\left(k_{x}, j_{y}, \sigma\right)=\sum_{m} e^{i m w t} u_{n, m}\left(k_{x}, j_{y}, \sigma\right)
$$

where as usual $\sigma=\uparrow, \downarrow$.

It is also interesting to consider the magnetization of each momentum value. The spin polarization is obtainable by

$$
\hat{m}\left(k_{x}, j_{y}\right)=\psi_{k_{x}, j_{y}, \uparrow}^{\dagger} \psi_{k_{x}, j_{y}, \uparrow}-\psi_{k_{x}, j_{y}, \downarrow}^{\dagger} \psi_{k_{x}, j_{y}, \downarrow}
$$


and the average spin polarization in the groundstate is given by

$$
\begin{array}{r}
m\left(k_{x}, j_{y}\right)=\sum_{n} \\
\left\{-\tilde{v}_{n}\left(-k_{x}, j_{y}, \uparrow\right) \tilde{v}_{n}^{*}\left(-k_{x} . j_{y}, \uparrow\right)\right. \\
\left.+\tilde{v}_{n}\left(-k_{x}, j_{y}, \downarrow\right) \tilde{v}_{n}^{*}\left(-k_{x} . j_{y}, \downarrow\right)\right\}
\end{array}
$$

\section{A. Charge currents}

The charge currents at the edges of the unperturbed Hamiltonian are well understood. If there is TRS the currents vanish and if TRS is broken the edge charge currents are finite (finite Chern number). We consider as examples a $d_{x}, d_{y}$ triplet superconductor and a $p+i p$ triplet superconductor. In the first case, if the magnetization vanishes, the system has TRS and vanishing charge edge currents in the topologically trivial phases. The charge current also vanishes in the $Z_{2}$ topological phase but the spin edge currents are non-vanishing. In the case of the $p+i p$ triplet superconductor there is no TRS and the charge edge currents are finite.

We consider in the case of the $d_{x}, d_{y}$ pairing a set of parameters $d=0.6, \Delta_{s}=0.1, d_{z}=0, \alpha=0.6$ and different values for the chemical potential and the magnetization. In the case of the $p+i p$ pairing we consider $d=0, \Delta_{s}=0.1, d_{z}=0.6, \alpha=0.1$.

In Fig. 6] we show results for the profile of the charge current as a function of $y$ for the various cases. We compare the unperturbed case with the perturbed one by considering that at $w=6$ it is enough to truncate the Hamiltonian matrix at $l=2$. To calculate the currents we sum over the states in the first Floquet zone. Also the results are for time $t=0$ or any multiple of the time period $T$.

The results for the $d_{x}, d_{y}$ pairing consider four points in the phase diagram: namely a trivial phase $\mu=-5, M_{z}=$ 0 (case $P_{1}$ with $C=0$ ), a $Z_{2}$ phase $\mu=-3, M_{z}=0$ (case $P_{2}$ with $C=0$ ), and two points in $Z$ topological phases $P_{3}$ with $\mu=-5, M_{z}=2$ (with $C=1$ ) and $P_{4}$ with $\mu=-1, M_{z}=2$ (with $C=-2$ ). The results for the $p+i p$ pairing consider $P_{5}$ with $\mu=-5, M_{z}=0$ (with $C=0$ ), $P_{6}$ with $\mu-2, M_{z}=0$ (with $C=2$ ), $P_{7}$ with $\mu=$ $-3, M_{z}=2$ (with $C=1$ ) and $P_{8}$ with $\mu=-1, M_{z}=2$ (with $C=-2$ ). These points are indicated in Fig. 1 .

In Fig. 6a we consider a periodic driving in the chemical potential with amplitude $\mu_{d}=1$ and in Fig. $6 \mathrm{~b}$ in the magnetization with the same amplitude. Focusing first on the unperturbed case we see that the current at the border vanishes for $P_{1}(C=0)$ and that for $P_{4}$ with $C=-2$ the current is larger at the edge, but decays faster with distance from the border with respect to the $P_{3}, C=1$ case. Adding the perturbations in the case of the trivial $P_{1}$ case, there is no charge current if driving the chemical potential but if one drives the magnetization there is a small current close to the edge. The
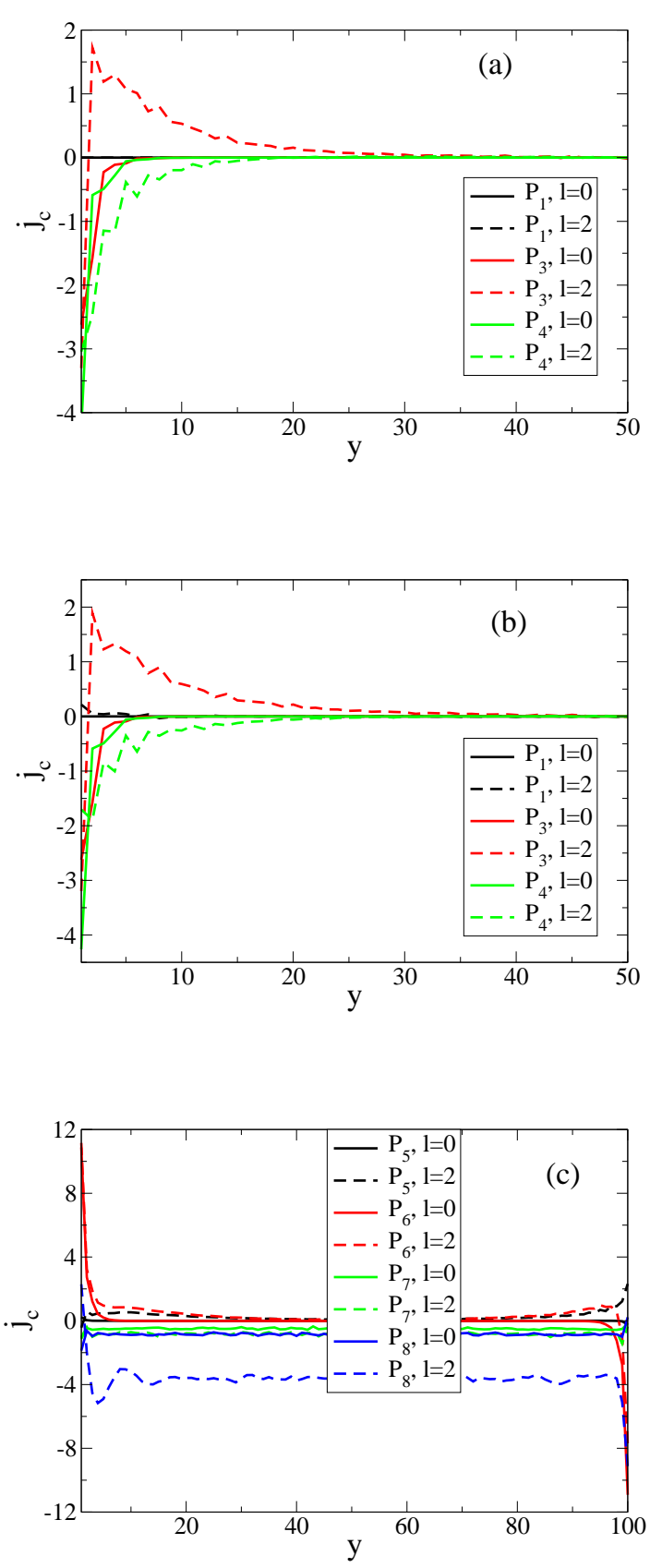

FIG. 6: (Color online) Charge current profile for $l=2$ for a $d_{x}, d_{y}$ superconductor for $P_{1}, P_{3}, P_{4}$ for (a) $\mu_{d}=1$ and (b) $M_{z d}=1$. Only one half of the system is shown since the current profile is anti-symmetric around the middle point. The system size is $N_{y}=100$. Charge current profile for $l=2$ for a $d_{z}$ superconductor for $P_{5}, P_{6}, P_{7}, P_{8}$ for (c) $\mu_{d}=1$. Note that the current profile has no symmetry around the middle point in some cases. The results for the $d_{x}, d_{y}$ pairing consider three points in the phase diagram: $\mu=-5, M_{z}=0$ (case $P_{1}$ with $C=0$ ), $P_{3}$ with $\mu=-5, M_{z}=2$ (with $C=1$ ) and $P_{4}$ with $\mu=-1, M_{z}=2$ (with $C=-2$ ). The results for the $p+i p$ pairing consider $P_{5}$ with $\mu=-5, M_{z}=0$ (with $C=0$ ), $P_{6}$ with $\mu-2, M_{z}=0$ (with $C=2$ ), $P_{7}$ with $\mu=-3, M_{z}=2$ (with $C=1$ ) and $P_{8}$ with $\mu=-1, M_{z}=2$ (with $C=-2$ ). These points are indicated in Fig. 1 . 


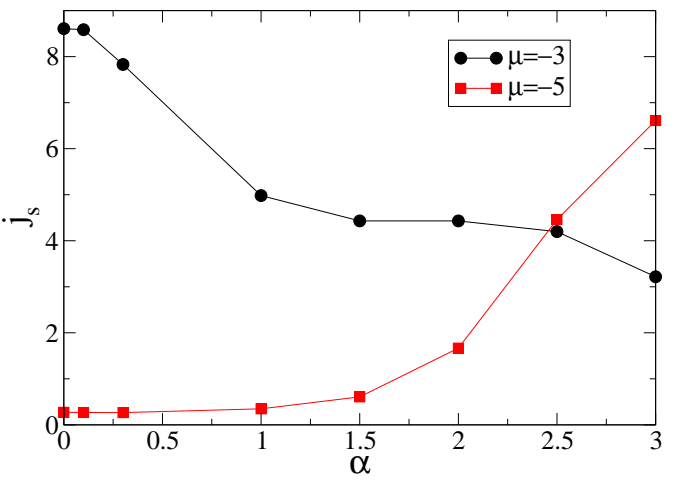

FIG. 7: (Color online) Influence of the spin-orbit coupling on the spin current in the unperturbed case. The parameters are $d=0.6, \Delta_{s}=0.1, d_{z}=0, M_{z}=0, \mu=-3,-5$. Increasing the spin-orbit in the topologically non-trivial phase is detrimental while in the topologically trivial phase, increasing the spinorbit coupling considerably increases the spin current.

effect of the perturbations on the topological cases is however quite significative, particularly in the case of $C=1$, with a current inversion with respect to the unperturbed case. A similar result has been found in the context of

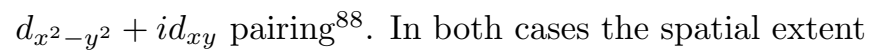
of the currents away from the border is considerably increased. However, we have checked that integrating the currents over $y$ from the edge to the middle point the total current is approximately the same. Also, we see that if the unperturbed state is topologically non-trivial the effect of the two types of perturbations is very similar.

In Fig. 6re we show results for the $p+i p$ pairing. Except for the case with $C=0$, the charge currents are finite in the unperturbed and perturbed cases. We note that, even for the $C=0$ case, there is a small charge current at the border, probably due to some finite size effects. In general, the driven chemical potential considered here increases the charge current. In the cases with zero magnetization the current decreases from the borders but at finite $M_{z}$ the current is finite throughout the whole system and is not symmetric around the middle point.

\section{B. Spin currents}

In this subsection we calculate the longitudinal spin currents at the edges of the system, both for the unperturbed and perturbed systems.

Consider first the unperturbed triplet superconductor. We start with the $d_{x}, d_{y}$ triplet superconductor in a trivial phase in zero magnetic field. In this regime there are no edge states and the system has a finite gap around zero energy. In Fig. 7 we consider the influence of the spinorbit coupling on the spin current in this trivial phase

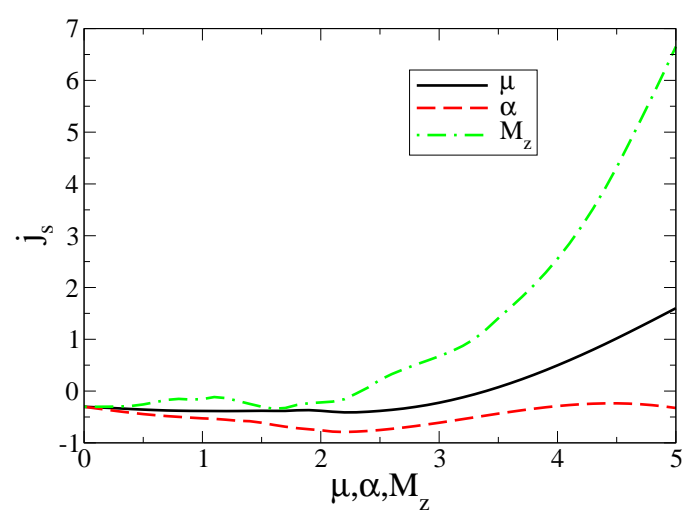

FIG. 8: Influence of intensity of periodic driving for the cases of $\mu_{d}, \alpha_{d}, M_{z d}$ on the spin current $(l=2)$. The parameters are $\mu=-5, d=0.6, \Delta_{s}=0.1, d_{z}=0, M_{z}=0, \alpha=0.6$ and the frequency of the periodic driving is $w=6$. Note the reversal of the direction of the spin current at small couplings. At weak coupling the increase of the spin current is linear. As the transition from weak to strong coupling occurs the spin current becomes non-linear. Changing the spin-orbit coupling has a small effect on the edge spin current while both changing the chemical potential and the magnetization have strong effects includind a change of the current direction.

and compare it with the case of a topological $Z_{2}$ phase, where there are edge states that, due to the spin locking, counterpropagate at each edge yielding a null charge current but a finite spin current. This is shown in the results for the case with $\mu=-3, M_{z}=0$. Increasing the spin-orbit coupling decreases the longitudinal spin current. An opposite effect is observed in the trivial phase for which, as the spin-orbit coupling increases, the spin current increases. At very small coupling the spin current is very small but non-vanishing, probably due to finite size effects. Even though there are no edge states the spin current is also carried out by the states in the continuum above and below the gap. When there are edge states these carry most of the current, but there is also a contribution from the other states in the continuum.

Consider now the perturbed cases. There are two parameters that characterize the perturbation: the frequency of the periodic driving and its amplitude. As discussed above, small frequencies require a large matrix to be diagonalized, particularly to determine the edge states. Very large frequencies are amenable to analytical solutions using the Magnus expansion and can be interpreted as a renormalization of the parameters of the original Hamiltonian, or by the addition of new terms allowed in the original unperturbed Hamiltonian. The cases treated here respect to intermediate values of the frequency, to allow for a simple truncation scheme and, at the same time, a resonance condition between bands that is most interesting in changing the topology of the system. Most results reported here are for a small to 

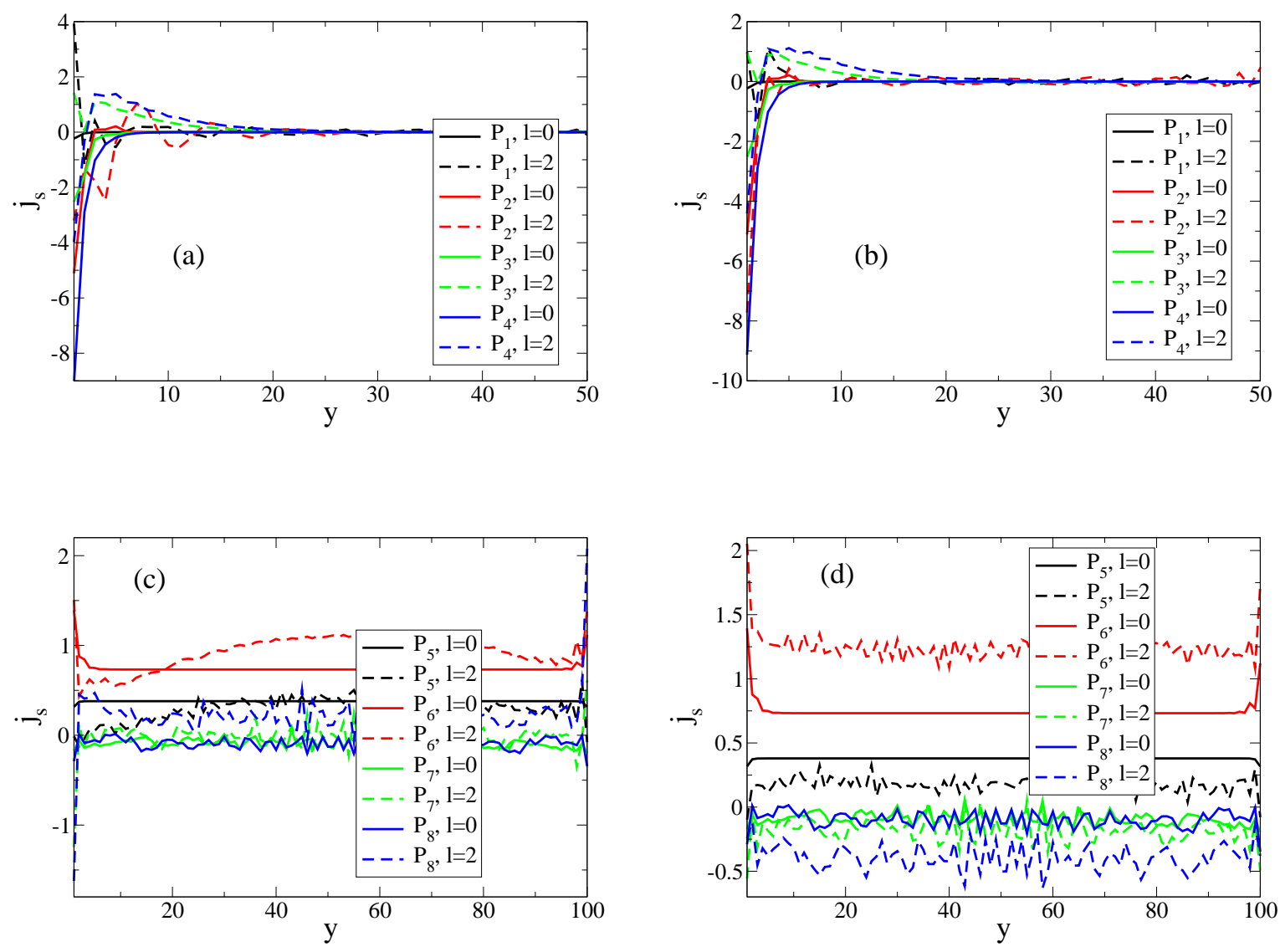

FIG. 9: (Color online) Spin current for the triplet superconductors $d_{x}, d_{y}((\mathrm{a}),(\mathrm{b}))$ and $d_{z}((\mathrm{c}),(\mathrm{d}))$ for the periodic drivings $\mu_{d}=1((\mathrm{a}),(\mathrm{c}))$ and $M_{z d}=1((\mathrm{~b}),(\mathrm{d}))$.
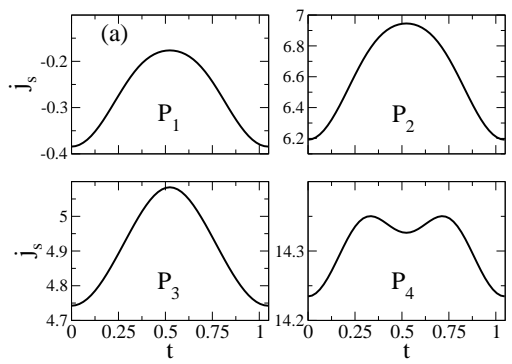
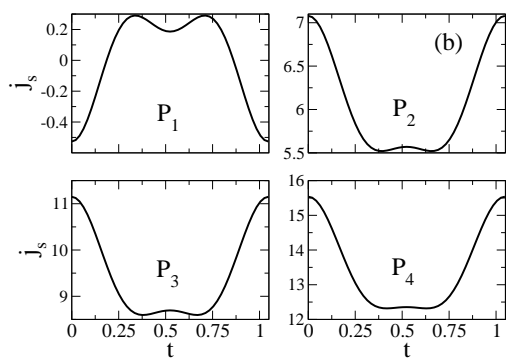
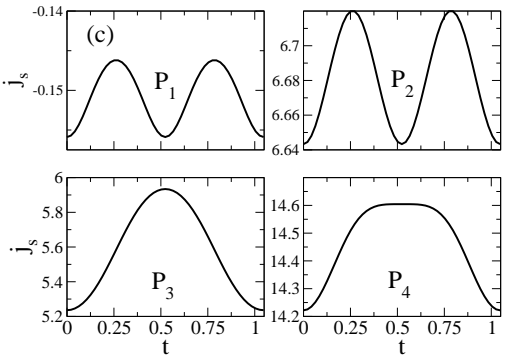

FIG. 10: Time evolution of the spin current for a $d_{x}, d_{y}$ triplet superconductor for the periodic drivings (a) $\mu_{d}$, (b) $\alpha_{d}$, and (c) $M_{z d}=1$ for $P_{1}, P_{2}, P_{3}, P_{4}$, respectively.

moderate amplitude. If the perturbation has a very small amplitude, it can be dealt with using perturbation theory. Very large amplitudes deviate considerably the system from its initial state. Therefore we have considered here moderate amplitudes. To illustrate the effect of the increase of the perturbation amplitude we show in Fig. 8 , for the same triplet superconductor, the effect of chang- ing the amplitude of a perturbation in the chemical potential, spin-orbit coupling and magnetization. As above for the charge currents, the results hold for time $t=0$ or for any multiple of the period $T$. The results for the spin current are obtained integrating the local spin current accross half the sample.

In all cases there is a sign reversal of the spin current. 
At small couplings, the behavior of the spin current $(l=$ 2 ) is approximately linear and the spin current does not change appreciably. Increasing the amplitude in the case of the spin orbit coupling, $\alpha_{d}$, does not affect much the spin current even though the response is no longer linear. On the other hand, the change in the chemical potential leads to a significant change of the spin current, changing back its direction for large enough values of the chemical potential, $\mu_{d}$, and the effect is even more pronounced in the case of the magnetization, $M_{z d}$. These results were obtained taking the unperturbed as a trivial phase with a very small spin current. The spin current is therefore significantly enhanced by the periodic driving.

In Fig. 9 we show results for $t=0$ (or a multiple of the period) of the spatial profile of the spin current for the triplet superconductors $d_{x}, d_{y}((\mathrm{a})$ and (b)) and $p+i p((\mathrm{c})$ and (d)) obtained changing the chemical potential, $\mu_{d}$, or the magnetization, $M_{z d}$. In the case of the first pairing symmetry, the spin current is antisymmetric around the middle point and so it is enough to consider half of the system. In the case of the $p+i p$ superconductor the current has no symmetry and so the full profile is shown, as before for the charge current.

The results are to some extent qualitatively the same as for the charge current, in the sense that the periodic driving induces a large spin current, if the unperturbed system is trivial, and enhances the spin current if the system is originally topological. The spin current oscillates, there is also reversal of direction due to the periodic driving, in the case of $C=-2$, and the spatial extent of the current profile increases. As for the unperturbed case, when there are edge states, the largest contribution to the current originates in them, but the continuum states also contribute.

In the case of the $p+i p$ superconductor the currents are spread along the whole system and in general are not symmetric around the middle point of the $y$ direction.

At arbitrary time values the currents oscillate. In Fig. 10 we show the time evolution of the spin current within one period, $T$, for the case of the $d_{x}, d_{y}$ triplet superconductor driven by the chemical potential, spin-orbit and magnetization. We consider as unperturbed states in each panel the trivial case $P_{1}$, the $Z_{2}$ topological point $P_{2}$, and the $Z$ topological phases $P_{3}$ and $P_{4}$. In the trivial phase, at short times, the perturbations decrease the spin current with respect to the initial time value (or any multiple of the time period). In the non-trivial phases the chemical potential and magnetization perturbations increase the spin currents at short times with respect to the initial time value while the spin-orbit coupling acts the opposite way.

\section{Spin polarization}

Associated with the spin currents in the topological phases, it has been shown that the eigenstates have non trivial spin polarizations that depend strongly on the
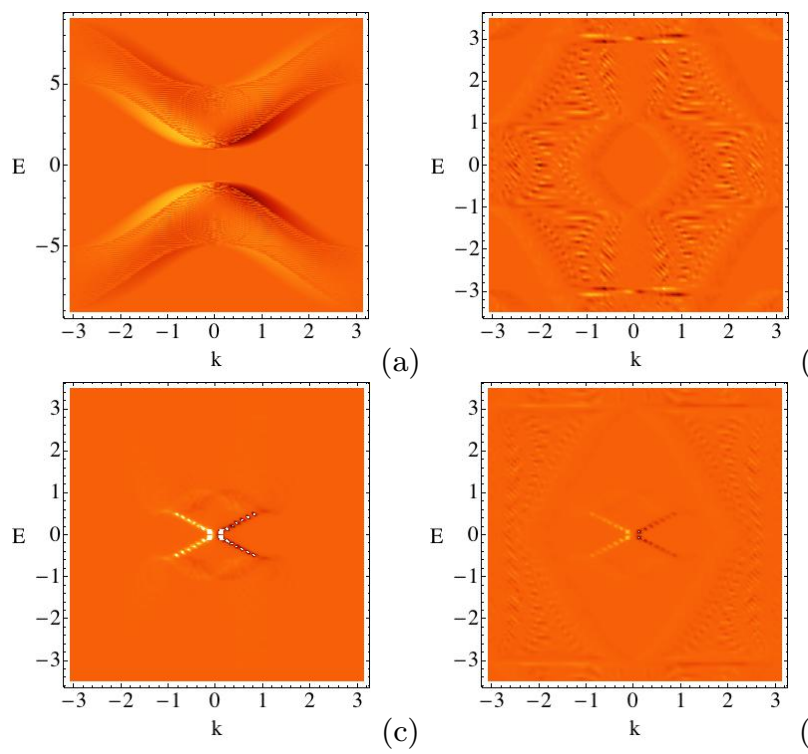

(a)

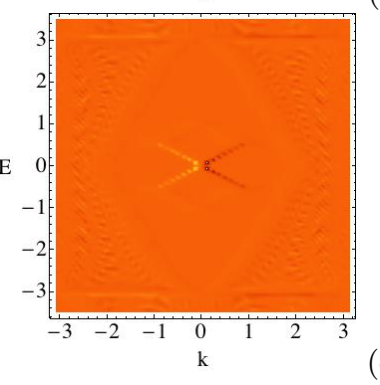

(b)

FIG. 11: (Color online) Spin polarization of unperturbed $(l=0)$ and perturbed $(l=2)$ system with $\mu_{d}=1$ for (a) and (b) $P_{1}$ (trivial case) and (c) and (d) $P_{2}\left(Z_{2}\right.$ case). In these cases $C=0$ and $M_{z}=0$. The maximal values of the spin polarizations are (a) $7.3 \times 10^{-5}$, (b) $1.9 \times 10^{-3}$, (c) $2.5 \times 10^{-2}$ and (d) $5.8 \times 10^{-3}$. The maximal value displayed in panel (c) has been reduced to 0.007 for better visualization.

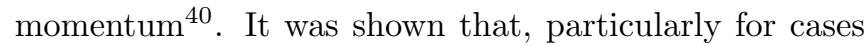
where one has flatbands, there is a strong polarization effect. Here we will look at the spin polarization of the Floquet states and compare with the unperturbed cases.

In Figs. 11 and 12 we show the spin polarization as a function of momentum for each quasi-energy state for the various examples, $P_{1}, P_{2}, P_{3}, P_{4}$ of the $d_{x}, d_{y}$ superconductor, both for the unperturbed and the perturbed cases with $l=2$.

The unperturbed cases in Fig. 11 show the nontrivial momentum distribution of the spin polarization for cases where $C=0$ and $M_{z}=0$. Summing over all momenta and states, the total polarization vanishes. The spin polarization shown in Fig. 11a has higher values at the edges of the gap and opposite signs as the momentum changes sign, due to the time reversal symmetry. Note, however, that the spin polarization is very small $\left(\sim 10^{-5}\right)$. In this regime of parameters the system is in a trivial phase.

Adding the periodic driving edge states are generated, as shown above, the spin polarization has higher values that are more uniformly spread over the various energy states and momenta, and with clearly higher values at the induced edge states at the border of the Floquet zone, again with opposite signs as the momentum changes sign since time reversal symmetry is not broken. However, the spin polarizations have a complex structure over the continuum. Panels (c) and (d) refer to a phase that shows edge states already at the level of the unperturbed Hamiltonian but with time reversal symmetry. The spin polarization is much stronger and sharply localized along the 

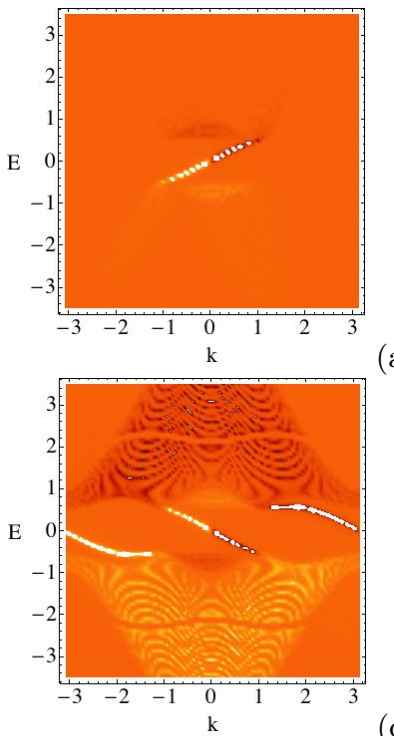

(a)
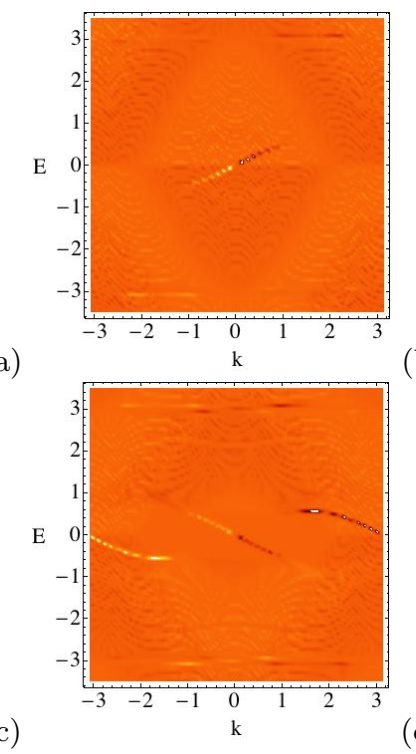

FIG. 12: (Color online) Spin polarization of unperturbed $(l=0)$ and perturbed $(l=2)$ system with $\mu_{d}=1$ for (a) and (b) $P_{3}(C=1)$ and (c) and (d) $P_{4}(C=-2)$. In these cases $C \neq 0$ and $M_{z} \neq 0$. The maximal values of the spin polarizations are (a) $3.8 \times 10^{-2}$, (b) $7.8 \times 10^{-3}$, (c) $4.7 \times 10^{-2}$ and (d) $8.3 \times 10^{-3}$. The maximal values of the panels have been reduced to 0.005 .

states that constitute the edge modes. Turning on the perturbation, the weight along the original edge states remains evident, but is reduced and there is a pile up of spin polarization along the new Majoranas generated at the edge of the Floquet zone. Note that, even though there is an alternancy of the polarization sign when the momentum sign is reversed, the two edge states are quite visible.

The results in Fig. 12 are different. They apply to cases for which the Chern number does not vanish, TRS is broken in the unperturbed system and there is a finite magnetization. The spin polarization is once again concentrated mostly along the edges but only one edge state is visible. The edge may be switched to the other one by reversing the sign of the magnetization. If the edge states are present in the unperturbed case, it is also clear that adding the time perturbaton decreases the polarization overall amplitude. Considering unperturbed topological phases, the spin polarization is highly concentrated on the edge states and less so in the perturbed case. In any case there is an enhancement with respect to the bulk states along the edge states, with either positive or negative spin polarizations in both branches of the edge states. However, in the presence of a magnetic field this symmetry is gone.

In the case of the $p+i p$ superconductor (not shown here) the states of the unperturbed Hamiltonian below the gap have a spin polarization that is large (and positive) for the negative momenta values but is considerably smaller for the positive momenta. The opposite happens for the positive energy states. Both cases correspond to

zero magnetization but the chiral nature is evident, both in the trivial case $C=0$ (expressed in the bulk states) and in the topologically non-trivial phase $(C=-2)$, (also in the edge states). This effect is somewhat washed out turning on the periodic driving, but both for the unperturbed and perturbed cases, only one edge state has a large spin polarization. In the perturbed case the edge state is immersed in the continuum. Nevertheless the spin polarization clearly singles it out.

\section{CONCLUSIONS}

A periodic driving on a topologically trivial system induces edge modes and topological properties. In this work we considered triplet and singlet superconductors subject to periodic variations of the chemical potential, spin-orbit coupling and magnetization in both topologically trivial and nontrivial phases and studied their influence on the charge and spin currents that propagate along the edges of the two-dimensional system. The most relevant case is the generation of the charge and spin edge currents due to the periodic driving in an otherwise trivial system. In some cases the generated currents are quite high and higher than in the unperturbed case providing a way to generate and control high spin currents with potential interest in the context of spintronics, particularly since some of the driving protocols involve electric or gate potential means, instead of a direct magnetic manipulation. Starting from a topologically non-trivial phase, the edge states originally present at zero energy are complemented by edge states at the edge of the Floquet zone at finite quasi-energies close to $\pm w / 2$. In general, the periodic driving smears the edge states, as shown particularly in the study of the spin polarization of the quasi-energy states, with a complex structure both in the unperturbed and perturbed cases. The chirality of the edge states is particularly seen when TRS is broken either by the presence of a finite magnetization or due to the intrinsic TRS breaking in a $p+i p$ triplet superconductor.

The transport signatures of edge states in driven topological insulators or due to the Floquet Majorana fermions in driven topological superconductors, involve the presence of leads to inject and collect charge or spin currents and therefore require the coupling of the system to outside reservoirs, with the associated issue of dissipation 80 . A calculation of the differential conductance has found a quantized conductance sum rule ${ }^{89}$, which generalizes the quantized zero-bias conductance contribution of the Majorana fermions when the system is not periodically driven. Here we neglected any couplings to external reservoirs. The charge currents calculated in this work may be detected considering the magnetic field they generate in their vicinity, as proposed in the context of unperturbed systems in Ref. ${ }^{82}$. Barring in mind the difficulty in detecting experimentally the edge currents, it may be challenging as well to detect the generated currents considered here. 
Discussions with Xiaosen Yang, Maxim Dzero, Antonio Garcia-Garcia and Pedro Ribeiro are acknowledged. Partial support in the form of a BEV by the CNPq at
$\mathrm{CBPF}$ is gratefully acknowledged as well as support from FCT through grant UID/CTM/04540/2013.
1 M. Z. Hasan and C. L. Kane, Rev. Mod. Phys. 82, 3045 (2010)

2 X.-L. Qi and S.-C Zhang, Rev. Mod. Phys. 83, 1057 (2011).

3 J. Alicea, Rep. Prog. Phys. 75, 076501 (2012).

${ }^{4}$ V. Mourik, K. Zuo, S. M. Frolov, S. R. Plissard, E. P. A. M. Bakkers, and L. P. Kouwenhoven, Science 336, 1003 (2012).

5 D.I. Tsomokos, A. Hamma, W. Zhang, S. Haas and R. Fazio, Phys. Rev. A 80, 060302(R) (2009).

${ }^{6}$ G.B. Halász and A. Hamma, Phys. Rev. Lett. 110, 170605 (2013)

7 L. D'Alessio and M. Rigol, arXiv:1409.6319

8 P.D. Sacramento, Phys. Rev. E 90, 032138 (2014).

9 E. A. Yuzbashyan, M. Dzero, V. Gurarie, and M. S. Foster, Phys. Rev. A 91, 033628 (2015).

10 D. Sen and S. Vishveshwara, Europh. Lett. 91, 66009 (2010).

11 M. S. Scheurer and A. Shnirman, Phys. Rev. B 88, 064515 (2013).

12 Matthew S. Foster, Maxim Dzero, Victor Gurarie, and Emil A. Yuzbashyan, Phys. Rev. B 88, 104511 (2013).

13 Matthew S. Foster, Victor Gurarie, Maxim Dzero, and Emil A. Yuzbashyan, Phys. Rev. Lett. 113, 076403 (2014).

14 A.Y. Kitaev, Phys.-Usp. 44, 131 (2001).

15 N. Read and D. Green, Phys. Rev. B 61, 10267 (2000).

16 M. Sato and S. Fujimoto, Phys. Rev. B 79, 094504 (2009).

17 Liang Fu and C. L. Kane, Phys. Rev. Lett. 100, 096407 (2008).

18 S. Nadj-Perge, I. K. Drozdov, B. A. Bernevig, and Ali Yazdani, Phys. Rev. B 88, 020407(R) (2013).

19 Stevan Nadj-Perge, Ilya K. Drozdov, Jian Li, Hua Chen, Sangjun Jeon, Jungpil Seo, Allan H. MacDonald, B. Andrei Bernevig and Ali Yazdani, Science 346, 602 (2014).

20 Yang Peng, Falko Pientka, Leonid I. Glazman, and Felix von Oppen, Phys. Rev. Lett. 114, 106801 (2015).

${ }^{21}$ Eugene Dumitrescu, Brenden Roberts, Sumanta Tewari, Jay D. Sau, and S. Das Sarma, Phys. Rev. B 91, 094505 (2015).

22 Jian Li, Titus Neupert, B. Andrei Bernevig, and Ali Yazdani, arXiv: 1404.4058

23 Ching-Kai Chiu, M.M. Vazifeh, and M. Franz, Europhy. Lett. 110, 10001 (2015).

24 Shusa Deng, Lorenza Viola, and Gerardo Ortiz, Phys. Rev. Lett. 108, 036803 (2012).

25 Shusa Deng, Gerardo Ortiz and L. Viola, Phys. Rev. B. 87, 205414 (2013).

26 M.A. Continentino, H. Caldas, D. Nozadze and N. Trivedi, Phys. Lett. A 378, 3340 (2014).

27 M. Dzero, K. Sun, V. Galitski and P. Coleman, Phys. Rev. Lett. 104, 106408 (2010).

28 M. Dzero, K. Sun, P. Coleman and V. Galitski, Phys. Rev. B 85, 045130 (2012).

29 Akira Furusaki, Masashige Matsumoto, and Manfred Sigrist, Phys. Rev. B 64, 054514 (2001).

30 P. D. Sacramento, M. A. N. Araújo, V. R. Vieira, V. K. Dugaev and J. Barnaś, Phys. Rev. B 85, 014518 (2012).
31 P.D. Sacramento, M.A.N. Araújo and E.V. Castro, Europhys. Lett. 105, 37011 (2014).

32 Sergey S. Pershoguba, Kristofer Björnson, Annica M. Black-Schaffer, and Alexander V. Balatsky, arXiv: 1505.01672

33 Abhiram Soori, Oindrila Deb, K. Sengupta, and Diptiman Sen, Phys. Rev. B 87, 245435 (2013).

34 Manisha Thakurathi, Oindrila Deb and Diptiman Sen, arXiv:1412.0072

35 R. Citro, F. Romeo, and N. Andrei, Phys. Rev. B 84, 161301(R) (2011).

36 Sho Nakosai, Jan Carl Budich, Yukio Tanaka, Bjrn Trauzettel, and Naoto Nagaosa, Phys. Rev. Lett. 110, 117002 (2013).

37 Saurabh Maiti, Manfred Sigrist, Andrey Chubukov, arXiv:1412.7439

38 Samuel Lederer, Wen Huang, Edward Taylor, Srinivas Raghu, and Catherine Kallin, Phys. Rev. B 90, 134521 (2014).

39 Wen Huang, Edward Taylor, and Catherine Kallin, Phys. Rev. B 90, 224519 (2014).

40 Andreas P. Schnyder, Carsten Timm, and P. M. R. Brydon, Phys. Rev. Lett. 111, 077001 (2013).

41 Andrew Peter Mackenzie and Yoshiteru Maeno, Rev. Mod. Phys. 75, 657 (2003).

42 C. Kallin, Rep. Prog. Phys. 75, 042501 (2012).

43 Jing Xia, Yoshiteru Maeno, Peter T. Beyersdorf, M. M. Fejer, and Aharon Kapitulnik, Phys. Rev. Lett. 97, 167002 (2006).

44 P. J. Curran, S. J. Bending, W. M. Desoky, A. S. Gibbs, S. L. Lee, and A. P. Mackenzie, Phys. Rev. B 89, 144504 (2014).

45 J. R. Kirtley, C. Kallin, C. W. Hicks, E.-A. Kim, Y. Liu, K. A. Moler, Y. Maeno, and K. D. Nelson, Phys. Rev. B 76, 014526 (2007).

46 Satoshi Kashiwaya, Hiromi Kashiwaya, Hiroshi Kambara, Tsuyoshi Furuta, Hiroshi Yaguchi, Yukio Tanaka, and Yoshiteru Maeno, Phys. Rev. Lett. 107, 077003 (2011).

47 Thomas Scaffidi and Steven H. Simon, arXiv:1410.6073.

48 Yasuhiro Tada, Wenxing Nie, and Masaki Oshikawa, Phys. Rev. Lett. 114, 195301 (2015).

49 G.E. Volovik, Pisma v ZhETF, 100, 843 (2014).

50 Yuujirou Amano, Masahiro Ishihara, Masanori Ichioka, Noriyuki Nakai, and Kazushige Machida, arXiv:1504.02836

51 A. Bermudez, D. Patanè, L. Amico and M.A. MartinDelgado, Phys. Rev. Lett. 102, 135702 (2009).

52 A. Bermudez, L. Amico and M.A. Martin-Delgado, New Journ. Phys. 12, 055014 (2010).

53 B. Bernevig, T. Hughes and S. Zhang, Science 314, 1757 (2006)

54 A.A. Patel, S. Sharma and A. Dutta, Eur. Phys. J. B 86, 367 (2013).

55 A. Rajak and A. Dutta, Phys. Rev. E 89, 042125 (2014).

56 M.-C. Chung, T.-H. Jhu, P. Chen, C.-Y. Mou and X. Wan, arXiv:1401.0433 
57 E. Perfetto, Phys. Rev. Lett. 110, 087001 (2013).

58 G. Kells, D. Sen, J. K. Slingerland, and S. Vishveshwara, Phys. Rev. B 89, 235130 (2014)

${ }^{59}$ R. Vasseur, J.P. Dahlhaus, and J.E. Moore, Phys. Rev. X 4, 041007 (2014).

${ }^{60}$ Benjamin M. Fregoso, Jan P. Dahlhaus, and Joel E. Moore, Phys. Rev. B 90, 155127 (2014).

61 Atanu Rajak, Tanay Nag, and Amit Dutta, Phys. Rev. E 90, 042107 (2014).

${ }^{62}$ Suraj Hegde, Vasudha Shivamoggi, Smitha Vishveshwara, and Diptiman Sen, arXiv:1412.5255.

63 Takashi Oka and Hideo Aoki, Phys. Rev. B 79, 081406(R) (2009).

64 N.H. Lindner, G. Refael and V. Galitski, Nat. Phys. 7, 490 (2011).

65 J.I. Inoue and A. Tanaka, Phys. Rev. Lett. 105, 017401 (2010); T. Kitagawa, E. Berg, M. Rudner and E. Demler, Phys. Rev. B 82, 235114 (2010)

${ }^{66}$ L. Jiang, T. Kitagawa, J. Alicea, A.R. Akhmerov, D. Pekker, G. Refael, J.I. Cirac, E. Demler, M.D. Lukin and P. Zoller, Phys. Rev. Lett. 106, 220402 (2011).

67 Qing-Jun Tong, Jun-Hong An, Jiangbin Gong, Hong-Gang Luo, and C. H. Oh, Phys. Rev. B 87, 201109(R) (2013).

68 Xiaosen Yang, arXiv:1410.5035

69 A. Poudel, G. Ortiz, and L. Viola, arXiv:1412.2639

70 D.E. Liu, A. Levchenko and H.U. Baranger, Phys. Rev. Lett. 111, 047002 (2013).

71 M. Thakurathi, A.A. Patel, D. Sen and A. Dutta, Phys. Rev. B 88, 155133 (2013).

72 M.S. Foster, V. Gurarie, M. Dzero and E.A. Yuzbashyan, Phys. Rev. Lett. 113, 076403 (2014).

73 Mark S. Rudner, Netanel H. Lindner, Erez Berg, and Michael Levin, Phys. Rev. X 3, 031005 (2013).

${ }^{74}$ Gonzalo Usaj, P. M. Perez-Piskunow, L. E. F. Foa Torres, and C. A. Balseiro, Phys. Rev. B 90, 115423 (2014).
75 A.P. Schnyder, S. Ryu, A. Furusaki and A.W.W. Ludwig, Phys. Rev. B 78, 195125 (2008; A.P. Schnyder, S. Ryu, A. Furusaki and A.W.W. Ludwig, in Advances in Theoretical Physics, edited by Vladimir Lebedev and Mikhail Feigel'man, AIP Conf. Proc. No. 1134 (AIP, Melville, NY, 2009), p. 10; S. Ryu, A.P. Schnyder, A. Furusaki and A.W.W. Ludwig, New J. Phys. 12, 065010 (2010).

76 J.K. Asbóth, B. Tarasinski and P. Delplace, Phys. Rev. B 90, 125143 (2014).

77 M. Benito, A. Gmez-Len, V. M. Bastidas, T. Brandes, and G. Platero. Phys. Rev. B 90, 205127 (2014).

78 Zi-bo Wang, Hua Jiang, Haiwen Liu, and X. C. Xie, arXiv: 1404.3029

79 Hossein Dehghani, Takashi Oka, and Aditi Mitra, Phys. Rev. B 90, 195429 (2014).

${ }^{80}$ Hossein Dehghani, Takashi Oka, and Aditi Mitra, Phys. Rev. B 91, 155422 (2015).

81 Christopher Grossert, Martin Leder, Sergey Denisov, Peter Hänggi, and Martin Weitz, arXiv:1407.0605

82 Jan P. Dahlhaus, Benjamin M. Fregoso, and Joel E. Moore, arXiv:1408.6811

83 M. Sigrist and K. Ueda, Rev. Mod. Phys. 63, 239 (1991).

84 P. A. Frigeri, D. F. Agterberg, A. Koga and M. Sigrist, Phys. Rev. Lett. 92, 097001 (2004).

85 D. Xiao, M.-C. Chang and Q. Niu, Rev. Mod. Phys. 82, 1959 (2010).

86 T. Fukui, Y. Hatsugai and H. Suzuki, J. Phys. Soc. Jpn. 74, 1674 (2005).

87 Takuya Kitagawa, Takashi Oka, Arne Brataas, Liang Fu, and Eugene Demler, Phys. Rev. B 84, 235108 (2011).

88 Bernd Braunecker, Patrick A. Lee, and Ziqiang Wang, Phys. Rev. Lett. 95, 017004 (2005).

89 Arijit Kundu and Babak Seradjeh, Phys. Rev. Lett. 111, 136402 (2013). 